\title{
Pediatric behavioral healthcare: the case for integrated services
}

\begin{abstract}
Pediatric practices often are unequipped with the necessary screening tools for mental illness in children, and children are typically referred out of the primary care setting to receive behavioral intervention. Integrated health care in pediatric settings involves the entire medical team working together to provide resources so medical care is more effective. Incorporating a behavioral health provider on the Primary Care team introduces an expert in the field of behavioral health. By including a behavioral health provider on the pediatric team, children and families can receive support for any behavioral or emotional concerns. Practices that use an integrated mental health approach report higher confidence in screening, more comprehensive and effective treatment, and a reduction in medical costs and work hours.
\end{abstract}

Keywords: pediatric, healthcare, behavioral health, mental health
Volume 9 Issue 6 - 2018

Diana Borrelli, Keith Klostermann
Department of Psychiatry, Medaille College,USA

Correspondence: Keith Klostermann, Department of Psychiatry, Medaille College, 18 Agassiz Circle, Buffalo, NY I42 |4, USA, Email kck35@medaille.edu

Received: July 23, 2018 | Published: December 20, 2018

\section{Pediatric behavioral healthcare: the case for integrated services}

According to a recent survey conducted by the National Alliance for Mental Illness (NAMI), only 34 percent of families that took their child to a pediatrician due to emotional and behavioral problems described their Primary Care Doctors as "knowledgeable" about mental illness. This finding is particularly troubling since the pediatrician's office is typically the first line of defense for concerned parents not understanding how they should handle their child's emotional or behavioral issues. Moreover, many parents may seek to manage these challenges by medicating their child and thus seek guidance and consultation about the possibility of beginning some form of psychotropic treatment. These concerns are not merely academic exercises but are reflected in the number of children who go undiagnosed or improperly treated due to unavailable screening tools and poor education in the mental health area among pediatric providers. Given that nearly seventy-five percent of children diagnosed with mental health conditions are seen in primary care settings, pediatricians need to have access to resources to provide intervention adequately. The purpose of this commentary is to review an integrated health care model and evaluate its usefulness with children.

\section{Mental health in pediatric practice}

Integrating mental health services in pediatric practice involves the inclusion of a behavioral heath provider (e.g., mental health counselor, marriage and family therapist) on the pediatric care team. The behavioral health provider should be a skilled child and family clinician and can provide an assessment of the patient's social, emotional, and psychological needs. Instead of referring a patient into the community to meet with a specialist, the pediatrician can screen and treat the patient and refer children and families to the behavioral health provider as needed to address any emotional, behavioral, or psychological concerns. The behavioral health provider may also screen the patient for the appropriate level of care and make referrals to more intensive services as needed. In fact, for practices with an embedded behavioral healthcare provider, the Pediatrician and behavioral health provider can coordinate treatment services to meet the patient's needs better. The use of integrated medical and behavioral health services provides the opportunity for a "warm handoff" in which the pediatrician can invite the behavioral health provider into the room to meet the family during appointments and explain the service with the hope of improving the family's engagement in behavioral health services. Since the family is familiar with the practice from attending medical appointments, there may be increased willingness among children and families to engage in treatment at the pediatrician's office rather than seeking mental health services in the community.

However, it is worth noting that merely adding a mental health counselor or family therapist to a pediatric team may be necessary but not sufficient. Many medical practices have adopted this approach only to see it falter because of the lack of clearly identified roles and confusion among nursing staff and providers about the proper workflow within the office. Integrated Health Care requires a collaborative effort between everyone on the healthcare team with an identified mission and vision about the scope of behavioral healthcare practice.

Duprey $^{1}$ developed a pediatric integrated health care implementation which identifies four primary phases that help guide successful integration and implementation efforts:

1. Education.

2. Logistics.

3. Develop and implement.

4. Workflow adjustment.

\section{Evaluate.}

The education phase provides structure in effectively conveying information about the integrated model with management, medical professionals, and general staff. The logistics phase examines how the integrated model works in the practice by evaluating specific needs and the manner in which the behavioral health provider meets these needs. In the develop and implement phase, a plan is developed to incorporate the integrated model into the practice. The workflow adjustment phase allows each practice to modify their plan as needed. Finally, in the evaluation phase, the newly implemented procedures and system are assessed for effectiveness and improvements made 
based on the results. Taken as whole, these phases create a systemic feedback loop for continuous quality improvement and refinement based on data gathered throughout the process.

\section{Outcomes}

The Children's Trauma Assessment Center (CTAC) at Western Michigan University focuses its work on traumatized children and adolescents involved in the child welfare, mental health, and judicial systems referred from across Michigan. ${ }^{2}$ The professionals involved with the CTAC recognized that many primary care practices operated in isolation, and that this separation from other health care professionals was negatively impacting the quality of screening and intervention for youth. The CTAC began using the integrated behavioral health model for their work with children and adolescents and found that this model worked well for their communities. The CTAC found that the primary care focus of this project demonstrated how personnel changes in a primary care pediatric practice could create a traumainformed and integrated primary care team (for further information see https://www.wmich.edu/traumacenter/michigan-genesee-countyappropriate-trauma-informed-screening-treatment-project).

A review of the effectiveness of integration implementation discovered variability in strategies required for integration. ${ }^{3}$ Theses researchers found that differences from practice-to-practice in integrated care pathways, governance models, integration of interventions, integrated care models, and health-care service integration. As a result, there may not be a standard process in transitioning to an integrated behavioral health model. According to Heyeres et al. ${ }^{3}$ the key to a successful implementation is to highlight the needs of the practice and how an integrated model will address those needs. Heyeres et al. ${ }^{3}$ also found encouraging results for the model, concluding health care providers feel much more confident in the care they provide due to the resources available through the model.

\section{Conclusion}

A large percentage of children with behavioral problems are seen by pediatricians, and the lack of mental health resources among these providers is becoming more evident. Pediatricians lack the relevant screening tools, and frequently outsource patients for intervention. Given the dearth of child psychiatrists, coupled with wait times of 4-6 months for first psychiatry appointments, pediatricians are increasingly being tasked with prescribing psychotropic medication to fill the gap until the first psychiatry appointment. Introducing a Behavioral Health Consultant onto the Primary Care team will allow more children to have their behavioral health needs identified and receive intervention for their needs. Instead of a pediatrician referring a patient to a specialist, they can screen and provide intervention to the patient with the guidance of the BHC. Integrating health care models requires a series of implementations steps, and these steps target and add resources to the specific needs of the clinical setting. Having an expert in the behavioral health field in pediatric practices provides many benefits including saving hours of work and medical costs. Schools that introduced BHCs and RNs as a preventative tool found a reduction in student absentees. Health care providers involved in the integrated model report more confidence in treating behavioral concerns due to the multidisciplinary nature of the treatment team. In conclusion, the benefits of integrated health care appear to benefit all parties involved - the health care providers and the patients.

\section{Acknowledgments}

None.

\section{Conflicts of interest}

The authors declare that there is no conflict of interest.

\section{References}

1. Duprey M. One location, one visit: A manual for integrating mental health services into a pediatric practice. Starfish Family Services. 2016.

2. Sloane MA. Implementation manual. Van Buren Transformational Health Initiative Project. 2016.

3. Heyeres M, Mccalman J, Tsey K, et al. The complexity of health service integration: A review of reviews. Front Public Health. 2016;4:1-8. 